\title{
HOMOCLINIC ORBIT FOR A FOURTH-ORDER SYSTEM
}

XIAOLEI REN

School of Mathematical Science

Nanjing Normal University

Nanjing, Jiangsu 210097

P. R. China

e-mail: rwxiaolei@163.com

\begin{abstract}
In this paper, homoclinic orbit of a fourth-order system will be concerned, several assumptions are made to prove a fourth-order system has a homoclinic orbit $q$ emanating from 0 where $q \in C^{4}\left(\mathbf{R}, \mathbf{R}^{n}\right)$. Under these assumptions, we can find the homoclinic orbit of the system by defining a function $I_{k}(q)$ via the Mountain Pass Theorem.
\end{abstract}

\section{Introduction and Main Results}

In this paper, we will deal with the existence of homoclinic orbit of the fourth-order system:

$$
-q^{(4)}+C q^{\prime \prime}-L(t) q+V_{q}^{\prime}(t, q)=0
$$

where $q \in \mathbf{R}^{n}, C$ is positive definite symmetric matrix, $L$ and $V$ satisfy:

2010 Mathematics Subject Classification: 37C60, 37B55, 34L30, 49J35, 49K15.

Keywords and phrases: homoclinic orbit, the Mountain Pass Theorem, critical point, periodic solutions.

Received September 17, 2019

(ㄷ) 2019 Scientific Advances Publishers 
(L) $L(t)$ is a continuous $T$-periodic matrix valued function and is a positive definite symmetric for all $t \in[0, T]$.

$\left(V_{1}\right) V \in C^{1}\left(\mathbf{R} \times \mathbf{R}^{n}, \mathbf{R}\right)$ is $T$-periodic in $t$.

$\left(V_{2}\right)$ There is a $\mu>2$ such that $0<\mu V(t, q) \leq\left(q, V_{q}^{\prime}(t, q)\right)$ for all $q \in \mathbf{R}^{n} \backslash\{0\}$.

$\left(V_{3}\right) V(t, q)=o\left(|q|^{2}\right)$ as $q \rightarrow 0$ uniformly for $t \in[0, T]$.

We say that a solution $q(t)$ of (1.1) is a homoclinic orbit if $q(t) \in C^{4}\left(\mathbf{R}, \mathbf{R}^{n}\right)$ such that $q(t) \rightarrow 0$ and $q^{\prime}(t) \rightarrow 0$ as $|t| \rightarrow \infty$.

Theorem 1.1. Assume $(L),\left(V_{1}\right)-\left(V_{3}\right)$ hold. Then (1.1) has a nonzero homoclinic orbit.

This paper is motivated by Rabinowitz [1], who has showed that under $(L),\left(V_{1}\right)-\left(V_{3}\right)$, the system

$$
q^{\prime \prime}-L(t) q+V_{q}^{\prime}(t, q)=0
$$

has a nontrivial solution $q \in C^{2}\left(\mathbf{R}, \mathbf{R}^{n}\right)$ satisfying $|q(t)|+\left|q^{\prime}(t)\right| \rightarrow 0$ as $|t| \rightarrow \infty$.

In Section 2, we will prove Theorem 1.1 using methods in [1].

\section{Proof of Theorem 1.1}

Similar to [1], for each $k \in N$, let $E_{k}=W_{2 k T}^{2,2}\left(\mathbf{R}, \mathbf{R}^{n}\right)$ with the norm:

$$
\|q\|_{k}^{2}=\int_{-k T}^{k T}\left[\left\|q^{\prime \prime}(t)\right\|^{2}+\left(C q^{\prime}(t), q^{\prime}(t)\right)+(L(t) q(t), q(t))\right] d t
$$


and let

$$
\begin{aligned}
I_{k}(q) & =\int_{-k T}^{k T}\left[\frac{1}{2}\left|q^{\prime \prime}(t)\right|^{2}+\frac{1}{2}\left(C q^{\prime}(t), q^{\prime}(t)\right)+\frac{1}{2}(L(t) q(t), q(t))-V(t, q)\right] d t \\
& =\frac{1}{2}\|q\|_{k}^{2}-\int_{-k T}^{k T} V(t, q) d t
\end{aligned}
$$

It is easy to check $I_{k} \in C^{1}\left(E_{k}, \mathbf{R}\right)$ and

$$
I_{k}\left(q^{\prime}\right) u=\int_{-k T}^{k T}\left[\left(q^{\prime}(t), u^{\prime \prime}(t)\right)+\left(C q^{\prime}(t), u^{\prime}(t)\right)+(L(t) q(t), u(t))-\left(V_{q}^{\prime}(t, q(t), u(t))\right)\right] d t .
$$

Lemma 2.1. If $I_{k}\left(q^{\prime}\right)=0$, then $q \in C^{4}\left(\mathbf{R}, \mathbf{R}^{n}\right)$ and $q=q(t)$ is a solution of (1.1).

Proof. If $q$ is a critical point of $I_{k}$, then

$$
\int_{-k T}^{k T}\left[\left(q^{\prime \prime}(t), h^{\prime \prime}(t)\right)+\left(C q^{\prime}(t), h^{\prime}(t)\right)+\left(L(t) q(t)-V_{q}^{\prime}(t, q(t)), h(t)\right)\right] d t=0,
$$

for all $h \in E_{k}$. Letting $\delta(t)=L(t) q(t)-V_{q}^{\prime}(t, q(t))$ means that $\int_{-k T}^{k T} \delta(t) d t=0$ since $\mathbf{R}^{n} \subset E_{k}$. So there is $x \in E_{k}$ satisfying $x^{\prime \prime}(t)=\delta(t)$ and

$$
\int_{-k T}^{k T}\left(\left(q^{\prime \prime}(t)-C q(t)+x(t)\right), h(t)\right) d t=0, \quad \forall h \in E_{k} .
$$

Thus, there is a constant $\xi \in \mathbf{R}^{n}$ such that $q^{\prime \prime}(t)-C q(t)+x(t)=\xi$ a.e. $x \in[-k T, k T]$. So

$$
q^{(4)}(t)-C q^{\prime \prime}(t)+L(t) q(t)-V_{q}^{\prime}(t, q(t))=0,
$$

since $\delta(t)=L(t) q(t)-V_{q}^{\prime}(t, q(t))$ is continuous. The proof is complete. 
In order to prove Theorem 1.1 as in [1], we will use the Mountain Pass Lemma.

Proposition 2.1. Let $E$ be a real Banach space and $I \in C^{1}(E, \mathbf{R})$ satisfy (PS). (PS) says whenever $I\left(u_{m}\right)$ is bounded and $I^{\prime}\left(u_{m}\right) \rightarrow 0$ as $m \rightarrow \infty$, the sequence $u_{m}$ possesses a convergent subsequence.

If further $I(0)=0$,

$\left(I_{1}\right)$ There exist constants $\rho, \alpha>0$ such that $\left.I\right|_{\partial B_{\rho}(0)} \geq \alpha$;

and

$\left(I_{2}\right)$ There exists $e \in E \backslash \bar{B}_{\rho}(0)$ such that $I(e) \leq 0$, then $I$ possesses a critical value $c \geq \alpha$ given by

$$
c=\inf _{g \in \Gamma} \max _{s \in[0,1]} I_{k}(g(s)),
$$

where

$$
\Gamma=\{g \in C([0,1], E) \mid g(0)=0 \text { and } g(1)=e\},
$$

$B_{\rho}(0)$ denotes an open ball of radius $\rho$ about 0 .

We will obtain a critical point of $I_{k}$ via the Mountain Pass Theorem. Moreover, the critical points of $I_{k}$ in $E_{k}$ are $2 k T$ periodic solutions of (1.1). First we need to prove $I_{k}(q)$ satisfies (PS). Recall that we say $I$ satisfied (PS) if that $I\left(u_{m}\right)$ is bounded and $I^{\prime}\left(u_{m}\right) \rightarrow 0$ imply that $u_{m} \rightarrow u$ for some $u \in E$ along a subsequence.

Lemma 2.2. $I_{k}(q)$ satisfies (PS).

Proof. To verify (PS), define first

$$
\|q\|_{\beta}=\left(\left\|q^{\prime \prime}\right\|_{L^{2}}^{2}+C\left\|q^{\prime}\right\|_{L^{2}}^{2}+(L(t) q, q)_{R}+\beta\|q\|_{L^{2}}^{2}\right)^{\frac{1}{2}},
$$


where $\beta>0$. Thus $\|\cdot\|_{\beta}$ is equivalent to $\|\cdot\|_{E_{k}}$. Suppose $q_{m}$ is a sequence in $E_{k},\left|I\left(q_{m}\right)\right| \leq K$ and $I^{\prime}\left(q_{m}\right) \rightarrow 0$. For $m$ large enough,

$$
\begin{aligned}
& \left|I^{\prime}\left(q_{m}\right) q_{m}\right| \\
& =\left|\left\|q_{m}\right\|_{\beta}^{2}-\beta\left\|q_{m}\right\|_{L^{2}}^{2}-\int_{-k T}^{k T}\left(q_{m}, V_{q}^{\prime}\left(t, q_{m}\right)\right) d \tau\right| \\
& \leq\left\|q_{m}\right\|_{\beta} .
\end{aligned}
$$

Therefore,

$$
\left|\int_{-k T}^{k T}\left(q_{m}, V_{q}^{\prime}\left(t, q_{m}\right)\right)_{R} d \tau\right| \leq\left\|q_{m}\right\|_{\beta}+\left\|q_{m}\right\|_{\beta}^{2}
$$

For $y \in R$ large enough, we have

$$
|y|^{2} \leq \mu\left(y, V_{q}^{\prime}\left(t, q_{m}\right)\right)
$$

Hence, by $\left(V_{2}\right)$ and (2.5)-(2.6) for a constant $\gamma$ independent of $m$, we have

$$
\begin{aligned}
\int_{-k T}^{k T} V\left(t, q_{m}\right) d \tau & \leq \frac{1}{\mu} \int_{-k T}^{k T}\left(q_{m}, V_{q}^{\prime}\left(t, q_{m}\right)\right) d \tau \\
& \leq \frac{1}{\mu}\left(\left\|q_{m}\right\|_{\beta}+\left\|q_{m}\right\|_{\beta}^{2}\right), \\
K & \geq \frac{1}{2}\left\|q_{m}\right\|_{\beta}^{2}-\frac{1}{2} \beta\left\|q_{m}\right\|_{L^{2}}^{2}-\int_{-k T}^{k T} V\left(t, q_{m}\right) d \tau \\
& \geq \frac{1}{2}\left\|q_{m}\right\|_{\beta}^{2}-\left(\frac{1}{2} \beta+\frac{1}{\mu}\right)\left(\left\|q_{m}\right\|_{\beta}+\left\|q_{m}\right\|_{\beta}^{2}\right)-\gamma
\end{aligned}
$$

Choosing $\beta \in\left(0,1-\frac{2}{\mu}\right)$, (2.7) shows $\left\|q_{m}\right\|_{E_{k}}$ is uniform bound, and then there is a subsequence of $q_{m}$ converges weakly in $E_{k}$ and strongly in $L^{\infty}$ 
to $q \in E_{k}$. The mean value of $q_{m}$ converges to the mean value of $\bar{q}$. By (2.4), we have $I\left(q^{\prime}\right)=q-\phi(q)$, where $\phi(q)$ is compact, hence $I\left(q^{\prime}\right) \rightarrow 0$, then $q_{m} \rightarrow \phi(\bar{q}),(P S)$ is verified. The proof is complete.

Lemma 2.3. If $(L)$ and $\left(V_{1}\right)-\left(V_{3}\right)$ hold, then $I_{k}$ defined in (2.2) possesses a critical point $q_{k}$ with the corresponding critical value $c_{k}$.

Proof. By (2.2), we have $I_{k}(0)=0$. By $\left(V_{2}\right)$, let $f(s)=V(t, s \xi)$; for any fixed point $\xi \in \mathbf{R}^{n}$, we have

$$
s f^{\prime}(s) \geq \mu f(s)=\mu V(t, s \xi)
$$

For $s \geq 1, s f^{\prime}(s) \geq \mu f(s)$. Integrate it in $[1, s]$, then $f(s) \geq s^{\mu} f(1)$. That is $V(t, s \xi) \geq s^{\mu} V(t, \xi)$, for this, we can get

$$
\begin{aligned}
V(t, \xi) & \leq V\left(t, \frac{\xi}{|\xi|}\right)|\xi|^{\mu}, \text { for } 0<|\xi| \leq 1, \\
& \geq V\left(t, \frac{\xi}{|\xi|}\right)|\xi|^{\mu}, \quad \text { for }|\xi| \geq 1 .
\end{aligned}
$$

Then there are constants $\alpha_{k}, \varepsilon_{k}>0$ such that

$$
\alpha_{k}\|q\|_{L^{\mu}[-k T, k T]} \leq\|q\|_{L^{x}[-k T, k T]} \leq \varepsilon_{k}\|q\|_{k} .
$$

Moreover by (2.1) and (2.8), we have

$$
I_{k}(\beta \varphi)=\frac{1}{2}\|\beta \varphi\|_{k}^{2}-\int_{-k T}^{k T} V(t, \beta \varphi) d t \leq \frac{1}{2} \beta^{2}\|\varphi\|_{k}^{2}-\int_{-k T}^{k T} V\left(t, \frac{\beta \varphi}{|\beta \varphi|}\right)|\beta \varphi|^{\mu} d t .
$$

Then there are constants $a_{1}, a_{2}>0$ such that

$$
I_{k}(\beta \varphi) \leq \frac{1}{2} \beta^{2}\|\varphi\|_{k}^{2}-a_{1}|\beta|^{\mu} \int_{-k T}^{k T}|\varphi| d t+a_{2}
$$

for all $\beta \in R$ and $\varphi \in E_{k} \backslash\{0\}$. By (2.1) and $\left(V_{3}\right), e=e_{k}$ and any $e_{k} \in E_{k} \backslash\{0\}$ shows $I_{k}\left(e_{k}\right) \leq 0$. Consequently by Proposition 2.1, $I_{k}$ 
possesses a positive critical value $c_{k}$ given by (2.2) and (2.3) with $E=E_{k}$ and $\Gamma=\Gamma_{k}$. Let $q_{k}$ denote the corresponding critical point of $I$ on $E_{k}$. The proof is complete.

Remark. Note that $q_{k} \neq 0$ since $c_{k}>0$.

Lemma 2.4. The $c_{k}$ and $q_{k}$ obtained in Lemma 2.3 are independent of $k$.

Proof. As in [1], we have $\varphi \in E_{1} \backslash\{0\}$ such that

$$
\begin{gathered}
\varphi( \pm T)=\varphi^{\prime}( \pm T)=0, \\
I_{1}(\varphi) \leq 0 .
\end{gathered}
$$

By (2.10), we can find $\phi$ satisfies (i), any sufficiently large multiple $\varphi$ of $\phi$ satisfies (i) and (ii), by Lemma 2.3 , let

$$
\begin{aligned}
e_{k}(t) & =\varphi(t) & & |t| \leq T \\
& =0 & & T \leq|t| \leq k T .
\end{aligned}
$$

Then by (2.8), we have $e_{k} \in E_{k} \backslash\{0\}$ and $I_{k}\left(e_{k}\right)=I_{1}\left(e_{1}\right) \leq 0$. Note also that $g_{k}(s)=s e_{k} \in \Gamma_{k}$ for all $k \in N$ and $I_{k}\left(g_{k}(s)\right)=I_{1}\left(g_{1}(s)\right)$. Therefore by $(2.2)$,

$$
c_{k} \leq \max _{s \in[0,1]} I_{1}\left(g_{1}(s)\right) \equiv M .
$$

So $c_{k}$ is independent of $k$ and (2.11) leads to a priori bounds for $q_{k}$. Since $I^{\prime}\left(q_{k}\right)=0$, by $\left(V_{2}\right)$,

$$
\begin{aligned}
c_{k} & =I_{k}\left(q_{k}\right)-\frac{1}{2} I_{k}^{\prime}\left(q_{k}\right) q_{k} \\
& =\int_{-k T}^{k T}\left[\frac{1}{2}\left(q_{k}, V_{q}^{\prime}\left(t, q_{k}\right)-V\left(t, q_{k}\right)\right] d t\right. \\
& \geq\left(\frac{\mu}{2}-1\right) \int_{-k T}^{k T} V\left(t, q_{k}\right) d t .
\end{aligned}
$$


Hence by (2.1) and (2.12), we have

$$
\begin{aligned}
\left\|q_{k}\right\|_{k}^{2} & =\int_{-k T}^{k T}\left[\left|q^{\prime \prime}(t)\right|^{2}+\left(C q^{\prime}(t), q^{\prime}(t)\right)+((L(t) q(t), q(t))] d t\right. \\
& =2 I_{k}(q)+2 \int_{-k T}^{k T} V(t, q) d t \\
& =2 c_{k}+\frac{4 c_{k}}{\mu-2} \\
& \leq 2 M\left(1+\frac{2}{\mu-2}\right) \equiv M_{1} .
\end{aligned}
$$

By (2.9), we have a $k$-dependent bound on $\left\|q_{k}\right\|_{L^{\infty}[-k T, k T]}$. The proof is complete.

Proof of Theorem 1.1. We can complete this theorem under the previous lemmas. By these lemmas, we obtain a critical point of $I_{k}$ and we know the critical points of $I_{k}$ in $E_{k}$ are periodic solutions of (1.1).

As in [1], for $q_{k} \in E_{k}, t, \tau \in[-k T, k T]$

$$
|q(t)| \leq q(\tau)|+| \int_{\tau}^{t} q^{\prime}(s) d s \mid
$$

We have

$$
\begin{aligned}
|q(t)| & \leq \int_{t-\frac{1}{2}}^{t+\frac{1}{2}}|q(t)| d \tau+\int_{t-\frac{1}{2}}^{t+\frac{1}{2}}\left|\int_{\tau}^{t} q^{\prime}(s) d s\right| d \tau \\
& \leq 2\left(\int_{t-\frac{1}{2}}^{t+\frac{1}{2}}\left(\left|q^{\prime}(\tau)\right|^{2}+|q(\tau)|^{2}\right) d \tau\right)^{\frac{1}{2}} \\
& \leq c\|q\|_{k}
\end{aligned}
$$


where $c$ depends on $L$. Hence

$$
\|q\|_{L^{\infty}[-k T, k T]} \leq c\|q\|_{k} .
$$

And so, (2.13) and (2.15) imply

$$
\left\|q_{k}\right\|_{L^{\infty}[-k T, k T]} \leq c M_{1}^{\frac{1}{2}} \equiv M_{2}
$$

Similarly, (2.16) will also hold if we replace $q_{k}$ with $q_{k}^{\prime}$. Because $q=q_{k}(t)$ is a solution of (1.1), $q_{k}$ is bounded in $C^{4}[a, b]$ for any $a<b$, and hence there is a subsequence of $q_{k}$ converging in $C_{\text {loc }}^{4}\left(\mathbf{R}, \mathbf{R}^{n}\right)$ to a solution $q$ of (1.1) satisfying

$$
\int_{-\infty}^{\infty}\left[\left|q^{\prime \prime}(t)\right|^{2}+\left(C q^{\prime}(t), q^{\prime}(t)\right)+(L(t) q(t), q(t))\right] d t<\infty .
$$

It follows that

$$
|q(t)|+\left|q^{\prime}(t)\right| \rightarrow 0 \text { as }|t| \rightarrow \infty .
$$

Now it is sufficient show $q(t) \neq 0$. By the inner product of (1.1) with $q_{k}$ and then integration by parts shows:

$$
\begin{gathered}
\int_{-k T}^{k T}\left(-q_{k}^{(4)}+C q_{k}^{\prime \prime}-L(t) q_{k}+V_{q}^{\prime}\left(t, q_{k}\right), q_{k}\right) d \tau=0 \\
\int_{-k T}^{k T}-\left|q_{k}^{\prime \prime}\right|^{2}-\left(C q_{k}^{\prime}, q_{k}^{\prime}\right)-\left(L(t) q_{k}, q_{k}\right)-V(t, q)+\left(V_{q}\left(t, q_{k}\right), q_{k}\right) d \tau=0 \\
\left\|q_{k}\right\|_{k}^{2}=\int_{-k T}^{k T}\left(q_{k}, V_{q}^{\prime}\left(t, q_{k}\right)\right) d t
\end{gathered}
$$

As in [1], set

$$
\begin{gathered}
Y(0)=0 \text { and for } s>0 \\
Y(s)=\max _{\substack{t \in[0, T] \\
|\xi| \leq s}} \frac{\left(\xi, V_{q}^{\prime}(t, \xi)\right)}{|\xi|^{2}} .
\end{gathered}
$$


By $\left(V_{1}\right)-\left(V_{2}\right), Y(s)>0$ if $s>0, Y \in C\left(\mathbf{R}^{+}, \mathbf{R}^{+}\right), Y$ is monotone nondecreasing and $Y(s) \rightarrow \infty$ as $s \rightarrow \infty$. Also by the definition of $Y$

$$
\frac{\left(q_{k}, V_{q}^{\prime}\left(t, q_{k}(t)\right)\right.}{\left|q_{k}(t)\right|^{2}} \leq Y\left(\left\|q_{k}\right\|_{L^{\infty}[-k T, k T]}\right) \quad \text { for all } t \in[-k T, k T] .
$$

Hence by (2.17) and (2.18),

$$
\begin{aligned}
\left\|q_{k}\right\|_{k}^{2} \leq & Y\left(\left\|q_{k}\right\|_{L^{\infty}[-k T, k T]}\right) \int_{-k T}^{k T}\left|q_{k}\right|^{2} d t \\
& \leq l_{0}^{-1} Y\left(\left\|q_{k}\right\|_{L^{\infty}[-k T, k T]}\right)\left\|q_{k}\right\|_{k}^{2},
\end{aligned}
$$

where $l_{0}>0$ satisfies $L(t) \geq l_{0} I_{2 n}, \forall t$. And

$$
Y\left(\left\|q_{k}\right\|_{L^{\infty}[-k T, k T]}\right) \geq l_{0}
$$

By the properties of $Y$, there is a $M_{3}>0$ such that

$$
\left\|q_{k}\right\|_{L^{\infty}[-k T, k T]} \geq M_{3} .
$$

Because $L$ and $V$ are $T$-periodic in $t$ and $q_{k}(t)$ is a $2 k T$-periodic solution, $q(t+j T), j \in[-k, k] \cap Z$ is also a solution of (1.1), and we may assume that the maximum of $\left|q_{k}(t)\right|$ occurs in $[0, T]$. Therefore if $q_{k}(t) \rightarrow 0$ in $C_{\text {loc }}^{1}$ along our subsequence,

$$
\left\|q_{k}\right\|_{L^{\infty}[-k T, k T]}=\max _{t \in[0, T]}\left|q_{k}(t)\right| \rightarrow 0 .
$$

Contrary to (2.19). The proof is complete.

\section{References}

[1] P. H. Rabinowitz, Periodic solutions of hamiltonian systems, Communications on Pure and Applied Mathematics 31(2) (1978), 157-184.

DOI: https://doi.org/10.1002/cpa.3160310203 
[2] V. C. Zelati and P. H. Rabinowitz, Homoclinic orbits for second order Hamiltonian systems possessing superquadratic potentials, Journal of the American Mathematical Society 4(4) (1991), 693-727.

DOI: https://doi.org/10.1090/S0894-0347-1991-1119200-3

[3] G.-W. Chen, Superquadratic or asymptotically quadratic Hamiltonian systems: ground state homoclinic orbits, Annali di Matematica Pura ed Applicata 194(3) (2015), 903-918.

DOI: https://doi.org/10.1007/s10231-014-0403-9

[4] P. H. Rabinowitz, Homoclinic orbits for a class of Hamiltonian systems, Proceedings of the Royal Society of Edinburgh Section A: Mathematics 114(1-2) (1990), 33-38.

DOI: https://doi.org/10.1017/S0308210500024240

[5] Y. Xu and M. Huang, Homoclinic orbits and Hopf bifurcations in delay differential systems with T-B singularity, Journal of Differential Equations 244(3) (2008), 582-598.

DOI: https://doi.org/10.1016/j.jde.2007.09.003

[6] A. R. Champneys and G. J. Lord, Computation of homoclinic solutions to periodic orbits in a reduced water-wave problem, Physica D: Nonlinear Phenomena 102(1-2) (1997), 101-124.

DOI: https://doi.org/10.1016/S0167-2789(96)00206-0

[7] A. Szulkin and W. M. Zou, Homoclinic orbits for asymptotically linear Hamiltonian systems, Journal of Functional Analysis 187(1) (2001) 25-41.

DOI: https://doi.org/10.1006/jfan.2001.3798

[8] G. A. Leonov, N. V. Kuznetsov and T. N. Mokaev, Hidden attractor and homoclinic orbit in Lorenz-like system describing convective fluid motion in rotating cavity, Communications in Nonlinear Science and Numerical Simulation 28(1-3) (2015), 166-174.

DOI: https://doi.org/10.1016/j.cnsns.2015.04.007

[9] G. A. Leonov, N. V. Kuznetsov and T. N. Mokaev, Homoclinic orbits, and self-excited and hidden attractors in a Lorenz-like system describing convective fluid motion, European Physical Journal-Special Topics 224(8) (2015), 1421-1458.

DOI: https://doi.org/10.1140/epjst/e2015-02470-3

[10] M. Izydorek and J. Janczewska, Homoclinic solutions for a class of the second order Hamiltonian systems, Journal of Differential Equations 219(2) (2005), 375-389.

DOI: https://doi.org/10.1016/j.jde.2005.06.029 
[11] A. R. Champneys, Y. A. Kuznetsov and B. Sandstede, A numerical toolbox for homoclinic bifurcation analysis, International Journal of Bifurcation and Chaos 6(5) (1996), 867-887.

DOI: https://doi.org/10.1142/S0218127496000485

[12] A. R. Champneys, Homoclinic orbits in reversible systems and their applications in mechanics, fluids and optics, Physica D: Nonlinear Phenomena 112(1-2) (1998), 158-186.

DOI: https://doi.org/10.1016/S0167-2789(97)00209-1 